\title{
LA PROTECTION ET LA POLITIQUE ÉCONOMIQUE ENVIRONNEMENTALES
}

\author{
Grace Ladeira Garbaccio ${ }^{1}$ \\ Instituto Brasileiro de Ensino, Desenvolvimento e Pesquisa (IDP)
}

Rocco Junior Flacco ${ }^{2}$

Università Roma Tre (UNIROMA3)

\section{RÉSUMÉ}

Cet article vise à présenter une réflexion sur la protection de l'environnement et la politique économique durable, en considérant certains éléments substantiels de la théorie des jeux appliquée aux réfugiés environnementaux, ainsi que sa rationalité procédurale analysée à la lumière du développement durable. En outre, les récentes catastrophes environnementales mettent en évidence les impacts économiques et la problématique des risques que la société mondiale endure. Ces impacts ne se limitent pas aux aspects économiques, mais reflètent également sur les marchés sociaux, de la santé, de la sécurité sanitaire, du travail et des finances. À travers une synthèse scientifique, cet article utilise la méthode de la recherche déductive et exploratoire, basée sur l'analyse des références d'articles et des études bibliographiques.

Mots-clés : développement durable ; politique économique environnementale ; protection de l'environnement ; réfugié environnemental ; théorie des jeux.

1 Docteure en Droit à l'Université de Limoges (UNILIM), avec le titre reconnu par l'Universidade Federal de Santa Catarina (UFSC). Master en Droit à l'UNILIM. Professeure du Programme Stricto Sensu du Master Académique en Droit et du Master Professionnel en Administration Publique à l'IDP. ORCID: https://orcid.org/0000-0002-0658-9472 / courriel: glgarbaccio@hotmail.com

2 Docteur en Droit Administratif au Département des Sciences Juridiques de l'Università di Roma «la Sapienza» (UNIROMA1). Professeur de Droit Administratif et de Droit Économique Public à l'UNIROMA3. Maître de conférences en Droit de la Santé au Master «Droit et gestion de la santé et services sociaux de santé, gestion des médicaments» à l'UNIROMA3 et au Master «Compétences et services juridiques en santé» à l' Università Cattolica del Sacro Cuore di Roma (UNICATT). ORCID: https://orcid.org/0000-0002-5929-1258 / courriel: rjflacco@gmail.com 


\section{A PROTEÇÃO E A POLÍTICA ECONÔMICA AMBIENTAIS}

\section{RESUMO}

O presente artigo visa apresentar uma reflexão acerca da proteção ambiental e da política econômica sustentável, considerando alguns elementos substanciais da teoria do jogos aplicada aos refugiados ambientais, bem como sua racionalidade procedimental analisada à luz da sustentabilidade. Além disso, as recentes catástrofes ambientais ressaltam os impactos econômicos e a problemática dos riscos que a sociedade mundial vem suportando. Tais impactos não se limitam ao aspecto econômico, sendo também refletido no social, na saúde, segurança sanitária, no mercado de trabalho e financeiro. Através de uma sintese científica, este artigo utiliza o método de pesquisa dedutiva e exploratória, com base na análise de referências de artigos e estudos bibliográficos.

Palavras-chave: desenvolvimento sustentável; política econômica ambiental; proteção do meio ambiente; refugiado ambiental; teoria dos jogos. 


\section{INTRODUCTION}

Canicules et changements climatiques, droit d'asile et mondialisation, rien à voir ? Nous ne sommes pas sûrs. A part l'instabilité politique et la croissance démographique, chaque année plus de personnes fuient les conditions hostiles de leur environnement.

L'opinion est informée par les médias d'un flux continu des catastrophes climatiques qui affectent le reste du monde : typhons, cyclones, sécheresses de grande ampleur, inondations diluviennes. Par contre, il est dangereux que la perception publique des conséquences physiques du changement climatique soit déformée : la nature des effets du changement climatique dont les incidences sont loin de se limiter aux phénomènes ci-dessus varie en fonction de l'ampleur de la hausse des températures et affecte de façon très inégale l'ensemble de la planète. Ainsi il faut prendre conscience que leurs conséquences ne sont donc pas immédiatement perceptibles, ni immédiatement descriptibles. D'autres phénomènes se développeront - l'arrivée d'espèces invasives, la création de milieux plus favorables à la propagation virale - et auront des conséquences profondes sur nos modes de vie.

L'écart historique entre le dernier trimestre de 2019 et le premier trimestre de 2020 a mérité une attention particulière dans le monde, en particulier en raison de l'émergence de situations qui sont presque complètement hors de contrôle de la normalité, sans réponses ni moyens de confinement efficaces. Catastrophes environnementales telles que les grands incendies dans les forêts d'Amazonie et d'Australie, ou la pandémie dévastatrice de coronavirus - dont l'origine probable aurait été la consommation humaine d'animaux sauvages -, qui s'est propagée de la Chine au reste du monde, causant des pertes de vies humaines et de pertes inestimables pour les économies nationales, ont conduit les pays touchés à prendre des mesures d'urgence désespérées, dont beaucoup, drastiques, sans perspective sûre de revenir à la normale à court terme. De telles situations permettent de reprendre les discussions sur les politiques transfrontalières visant à équilibrer l'équation " environnement / économie ", avec des problèmes à résoudre par les États, en tant que membres d'une communauté internationale.

Si le risque n'est pas immédiatement perceptible, si son ampleur n'est pas encore quantifiable, nous pouvons au minimum dire qu'il est certain. Nous aurions tort d'oublier qu'à l'échelle des temps le climat tempéré qui 
a permis l'essor de l'Europe n'est qu'une rareté au milieu de périodes géologiques caractérisées par une très grande sécheresse. C'est tout le climat tempéré sur la planète qui est en cause.

Les changements climatiques peuvent affecter la biodiversité d'une région tempérée ou tropicale, etc., par l'invasion de certaines espèces végétales et animales et induire ainsi une migration populationnelle. L'article vise à présenter également un champ scientifique d'une manière complète et claire, basé sur la méthode de recherche déductive et exploratoire à partir de l'analyse des references d'articles scientifiques et d'études bibliographiques. Passons au traitement du cas des réfugiés environnementaux.

\section{L'ANALYSE DE LA PRISE DE DÉCISION DES RÉFUGIÉS ENVIRONNEMENTAUX AU REGARD DE LA THÉORIE DES JEUX}

Théoriquement, les réfugiés écologiques n'existent pas. En tout cas, on ne leur reconnaît pas le titre de « réfugiés ", réservé, selon la Convention de Genève de 1951, à ceux qui doivent quitter leur pays à cause de persécutions liées à leur race, leur religion, leur nationalité ou leurs opinions politiques. Sans cette reconnaissance, ils ne peuvent espérer une admission de responsabilité de la part de la communauté internationale ni une prise en charge.

Des catastrophes ${ }^{3}$ provoquées par la réaction de la nature face aux comportements humains instaure une situation de déplacement des êtres humains : il quittent leurs maisons ou leurs pays. Cette migration engendre des déséquilibres aux lieux de départ ainsi que celui d'arrivée : les relations homme/nature, société/espace, ressource/population.

Si beaucoup fuient les conditions précaires de leur environnement, c'est parce qu'ils/elles n'ont pas le choix. Comme le souligne avec justesse Gallais (1994, p. 7) «l'homme est toujours victime de la catastrophe dont il aura le plus souvent, directement ou indirectement, accru le risque ».

Cependant les déplacements de population ne sont pas un phénomène récent. La nouveauté demeure sur le risque lié à l'ampleur du mouvement de population. Celui-ci est crée par la combinaison de plusieurs facteurs : épuisement des ressources, destruction irréversible de l'environnement et croissance démographique, etc.

3 Par exemple le nuage radioactif de Tchernobyl, les inondations près du barrage des Trois Gorges en Chine, la submersion annoncée de l'archipel de Tuvalu en Polynésie, etc. 
Il faut souligner la différence entre réfugiés et émigrants. Le critère différencié est attaché à la liberté. Un émigré a choisi cette situation, le réfugié non. Nous pouvons évoquer dans l'avenir le conditionnement de ces réfugies à partir de la limitation de leur nombre au travers des quotas de réfugiés d'un pays vers l'autre.

Ainsi, il faut analyser la prise de décision d'un émigrant différemment de la prise de décision d'un réfugié écologique. La première est autonome et inconditionnée, pendant que l'autre est obligée et conditionnée.

L'assistance écologique est un principe du Droit international de l'environnement (DIE), est une coopération. L'assistance est liée au droit de l'homme, c'est un devoir de la communauté internationale. Mais le DIE est insuffisant pour protéger les réfugiés. Il invite à reconnaître et à proclamer un statut international des réfugiés écologiques permettant d'assurer la protection de cette catégorie à part entière de réfugié. Il invite aussi à agir préventivement contre les différentes causes à l'origine des situations de réfugiés écologiques : catastrophes, mauvaises gestions et planifications des ressources naturelles, les changements climatiques, etc. Il invite aussi à élaborer des politiques à long terme favorisant la protection des réfugiés écologiques.

Au-delà du phénomène des réfugiés environnementaux, nous constatons la mise en œuvre d'une rationalité procédurale qui suppose une rationalité basée sur des objectifs intermédiaires.

\section{LA MISE EN GEUVRE D'UNE RATIONALITÉ PROCÉDURALE ET LA THÉORIE DES JEUX}

C'est l'articulation des indicateurs énergétiques et de la théorie du capital qui nous amène à l'arbre de la durabilité dynamique : tout choix politique concernant le développement soutenable/durable est confronté à l'incertitude, l'irréversibilité et la complexité ou encore à la multi dimensionnalité des critères d'ordre écologique, économique et social. Comme l'exposent Faucheux et Noel (1995, p. 311) «c'est l'interaction entre ces éléments qui constitue un mélange explosif pour la prise de décision. Ceci présuppose un paradigme de la rationalité économique plus large que celui de l'économie orthodoxe, et appelé rationalité procédurale ».

Une interprétation économico écologique du développement durable : selon Ruth (1994) l'intégration des concepts centraux de l'économie, l'écologie et la thermodynamique est fondamentale pour « développer une 
extension des modèles économiques qui puisse rendre compte explicitement d'un certain nombre d'interactions économie/environnement prenant place sous forme d'échange de matière et d'énergie entre les deux systèmes (économiques et écologiques) » (FAUCHEUX; NOEL, 1995, p. 310). Selon ces auteurs, il est d'un intérêt vital pour les générations présentes et futures de connaître les limites imposées aux processus économiques par l'écosystème dans lequel est inclus le système économique.

Les concepts centraux de l'économie sont : les coûts d'opportunité, la substitution, les préférences temporelles. Les concepts centraux de la thermodynamique sont : la définition du système et de ses limites, l'évaluation des flux de matière et d'énergie au travers de ses limites au moyen des lois thermodynamiques, la distinction de systèmes ayant des niveaux d'ordres différents. Les concepts centraux de l'écologie sont : les cycles de la matière, les flux de l'énergie, la complexité des interactions système/ environnement qui s'expriment dans les processus de feed-back entre les composantes des écosystèmes.

La mise en œuvre d'une rationalité procédurale peut impliquer le remplacement d'un objectif global non mesurable par des objectifs intermédiaires, voire des sous objectifs intermédiaires dont la réalisation peut être observée et mesurée. Dans un schéma de rationalité procédurale, on peut, à partir de l'objectif global qu'est la durabilité du développement et qui s'avère non mesurable directement, déterminer des objectifs intermédiaires à cette durabilité.

Les objectifs intermédiaires peuvent être identifiés aux trois filtres : écologique, social et économique. Chacun de ces objectifs intermédiaires peut être lui-même éclaté en plusieurs sous objectifs intermédiaires prenant la forme de normes à respecter. Ainsi, une première étape consiste à déterminer de multiples sous objectifs intermédiaires qui sont irréductibles et qui doivent être simultanément considérés.

Le Protocole de Kyoto a déterminé des objectifs globaux environnementaux face aux développements économique et social à partir d'objectifs intermédiaires (des objectifs régionaux, le cas de l'Union européenne, et des objectifs locaux, par pays) concernant le niveau de réduction des émissions de GES au niveau de 1990. Les sous objectifs ont été établis par chaque région ou par chaque pays. Lors de la discussion du protocole, l'UE a négocié une provision - la bulle européenne - qui permet à ses Etats de remplir conjointement l'objectif global de 8\% des émissions de GES des pays du l'Annexe B. 
Cet objectif a été décliné ensuite dans les différents Etats membres, en fonction des situations nationales. La France, notamment du fait d'une production d'électricité principalement assurée par des centrales nucléaires et hydrauliques, peu émettrices de GES, s'est ainsi vu assigner un objectif de stabilisation de ses émissions à 1990 . Or compte tenu de l'évolution tendancielle constatée en 2002 (une politique de « laisser faire » verrait croitre d'environ 10\% les rejets de GES entre 1990 et 2010), atteindre un tel objectif suppose de réduire de 54 millions de tonnes équivalent de $\mathrm{CO} 2$ (Mtéq CO2) nos émissions de GES par an à l'horizon 2010. Soit l'équivalent de $13 \%$ des rejets de GES estimés en 2003 (FRANCE, 2020).

Il y a la méthode qui définit des choix satisfaisants au lieu des choix optimaux : dans ce cadre de rationalité procédurale, le décideur ne retient pas la solution optimale, mais choisit la solution qui lui paraît la plus satisfaisante au regard de différents impératifs, qu'ils soient écologiques, économiques, sociaux ou autres. Le recours à ce principe montre que l'exigence de durabilité n'est pas forcément l'inscription dans une démarche d'optimisation et qu'elle doit plutôt correspondre à une norme minimale de satisfaction (FAUCHEUX; NOEL, 1995, p. 312) 4 . Le point optimum établi pour la France par le protocole a été de maintenir de manière constante ses émissions de GES au niveau de 1990, par contre la France s'est fixé une réduction de 54 millions de tonnes équivalent de $\mathrm{CO} 2$.

Nous présentons ici un instrument de prise de décision, basé sur un tel principe pour tester les trajectoires de « soutenabilité » d'une nation. Avec une perspective dynamique du développement soutenable, il est nécessaire d'introduire les compensations offertes par les balances externes, le progrès technique et les possibilités de substitution entre le capital naturel et le capital manufacturé. Ces éléments sont introduits dans un arbre de « soutenabilité » et on obtient un processus de prise de décision séquentiel et itératif, articulant les indicateurs énergétiques et les indicateurs issus de la théorie du capital.

Quelques indicateurs énergétiques permettent la réalisation partielle de deux des sous objectifs du développement soutenable à savoir la « soutenabilité » économique et écologique. Le premier, le surplus eMergétique, représente un sous objectif intermédiaire de reproduction des ressources naturelles.

4 Ajoutons que le principe de satisfaction est séquentiel, il doit spécifier les mécanismes suivants : un mécanisme de comparaison entres les actions et le niveau désiré qui assure une classification séquentielle entre des actions satisfaisantes et des actions non satisfaisantes ; un mécanisme d'endogénéisation partielle du niveau désiré qui s'ajuste de lui-même quand le mécanisme de comparaison précédent ne génère pas immédiatement la solution satisfaisante. 
Il consiste à normaliser l'ensemble de ressources naturelles par leur transformation solaire et à mesurer l'apport en ressources de l'environnement au travers de la totalité de l'interface économie/environnement. Le surplus eMergétique national, de l'anglais National EMERGY surplus (NES), est donné comme la différence entre la quantité eMergétique produite avec les ressources naturelles à l'intérieur d'un pays et la quantité eMergétique consommé par de dernier. Le surplus eMergétique disponible, de l'anglais Available EMERGY Surplus (AES), est défini comme la différence entre la quantité eMergétique disponible et la quantité eMergétique consommée par une économie ouverte (FAUCHEUX; NOEL, 1995, p. 312).

Un développement durable écologique nécessite que NES soit égal ou supérieur à zéro. Pour satisfaire une telle condition, les taux de prélèvement de toutes les ressources naturelles ne doivent jamais être supérieurs aux taux de renouvellement et de reconstitution de ces dernières. Ainsi NES mesure donc la marge disponible pour un développement potentiel dans l'extraction des ressources et il permet la mesure cohérente en termes physiques de deux contraintes écologiques traditionnelles selon la perspective de la « soutenabilité » forte. Si NES est égal ou inférieur à zéro, cela signifie que la consommation des ressources naturelles est plus grande que le taux de renouvellement de ces dernières et que l'économie n'est plus durable d'un point de vue écologique.

Par contre, l'AES égal ou supérieur à zéro est un critère plus faible de durabilité écologique car le développement d'une économie s'effectue au dépend des autres (si un pays exporte des ressources naturelles vers l'économie nationale, il peut avoir NES égal ou supérieur à zéro et AES égal ou inférieur à zéro, de telle sorte qu'il devient insoutenable.

Le second, le surplus énergétique, le sous objectif intermédiaire de rendement énergétique du système économique, sont des indicateurs de l'efficacité énergétique d'un système économique. La procédure d'évaluation mesure l'énergie en fonction de sa capacité à générer un travail mécanique, ce qui constitue la mesure la plus intéressante de la qualité énergétique d'un point de vue économique. Une telle évaluation peut être utilisée pour quantifier ce qui est qualitativement appréhendé comme la détérioration énergétique, c'est-à-dire la dégradation thermodynamique d'un système donné. L'objectif de durabilité implique que le système économique puisse connaître une reproduction étendue. La poursuite du développement économique sur une base élargie impose, du point de vue énergétique, un surplus énergétique permanent.

Le surplus énergétique national, de l'anglais National Exergy Surplus (NES), représente la valeur énergétique dans une période de temps donnée, 
comme la différence entre la valeur énergétique (contenu libre) des inputs disponibles pour la production et la quantité d'énergie dissipée dans un processus de consommation ou de production.

Le surplus énergétique disponible, de l'anglais Available Exergy Surplus (AES), est identifié comme la différence entre la valeur énergétique des inputs disponibles pour la production (les stocks à l'intérieur de l'économie, les stocks importés et le flux d'énergie libre à l'intérieur de l'économie) et la quantité d'énergie dissipée dans le processus économique (production, consommation ou exportation).

L'indicateur NES permet de voir si un développement économique peut continuer sur une base élargie ou non ${ }^{5}$.

Dans la proposition intermédiaire de sous objectifs définis par des procédures d'évaluation énergétiques, l'interface entre l'économie et l'environnement a été réduit à un mouvement : celui de l'extraction des ressources naturelles et celui du rejet de pollution dans la biosphère. S'il est bien certain que l'essentiel des relations entre économie et environnement passe par ces deux dimensions, il ne faut pas oublier qu'existent d'autres dimensions de la durabilité écologique telles que l'espace ou la biodiversité. Pour de tels aspects de la durabilité écologique, se pose bel et bien un problème d'absence de mesures physiques homogènes qui empêche l'agrégation au niveau macroéconomique.

Pour comprendre l'arbre de durabilité, il faut examiner toutes les possibilités qu'un pays a de retrouver un sentier de durabilité potentielle. Ces éléments donnent un sens au concept de processus de décision.

En effet, le décideur peut tester différentes politiques de développement soutenable car plusieurs sentiers de développement soutenable sont maintenant possibles grâce à l'introduction des possibilités de compensation offertes par les balances externes, le progrès technique et partiellement par les élasticités de substitution (FAUCHEUX; NOEL, 1995, p. 315-316).

Ainsi, d'autres indicateurs peuvent être définis, tels l'Emergy External Balance (EME) comme la différence entre les exportations et les importations de capital naturel exprimée en termes eMergétiques ; l'Exergy External Balance (EXEB) comme la différence entre les exportations et les importations de capital naturel exprimée en termes énergétiques.

$5 \mathrm{Si}$ NES $<0$, cela signifie qu'il y a une énergie mécanique insuffisante dans le pays pour permettre une reproduction économique. Si NES =0, tout dépend de l'état initial de l'économie en question. Si elle a déjà atteint un haut degré de maturité et si NES $>0, \mathrm{NES}=0$ indique une situation stationnaire où seule une reproduction simple sans accumulation (croissance) semble possible. Si NES $>0$, le système économique produit un surplus qui peut être utilisé pour entreprendre une "reproduction élargie » de telle sorte que le développement est soutenable (FAUCHEUX; NOËL, 1995. p 314). 
Un pays peut satisfaire ses besoins en eMergie et en exercice et obtenir un surplus eMergétique, ainsi qu'un surplus énergétique grâce au commerce international, éventuellement aux dépens d'un autre pays. Pour ce dernier, la valeur des deux indicateurs décroîtra corrélativement et peut devenir négative.

En conséquence, nous passons à l'analyse des éléments économiques internes et externes en étant comme objectif approfondir ce sujet vers le développement durable.

\section{DES ÉLÉMENTS ÉCONOMIQUES INTERNE ET EXTERNE VERS LE DÉVELOPPEMENT DURABLE}

« Les conditions et la mesure de la « soutenabilité » ne sont pas sans difficultés significatives, mais en utilisant les enseignements existants on peut faire de réels progrès dans cette voie » (FAUCHEUX; NOEL, 1995, p. 328).

Nous pouvons dire qu'il n'existe pas une mais plusieurs conceptions économiques de l'environnement et des ressources naturelles et autant d'approches diverses du développement durable. Certes, leurs partisans respectifs ont tendance à les présenter comme exclusives les unes des autres. Or «l'économie écologique » prouve par sa démarche qu'une certaine complémentarité est possible. Par exemple, en recourant à l'utilisation conjointe des enseignements sur la gestion des systèmes naturels issus des analyses néo-classiques et de la conception " conservatrice » prônant le maintien du capital naturel critique, on peut développer, au-delà des disciplines économiques et écologiques conventionnelles, une véritable synthèse intégrée. En ce qui concerne l'éthique, l'économie écologique peut s'ouvrir aux différentes conceptions de l'être humain et aux diverses approches de la justice et de la solidarité sociale. Il s'agit alors d'abandonner l'idée de construire une économie des ressources naturelles et de l'environnement afin de reconstruire une économie pour les ressources naturelles et l'environnement, tout en proposant des moyens pour parvenir à cette fin.

Ainsi que l'écrit Costanza (1991), « pour atteindre la « soutenabilité » globale, nous devons cesser de penser les objectifs économiques et écologiques comme étant conflictuels $[\ldots]$. Nous devons développer une économie écologique qui aille au-delà des distinctions économiques et écologiques conventionnelles pour aller dans le sens d'une véritable synthèse intégrée (FAUCHEUX; NOEL, 1995, p. 330). 


\subsection{LA POLITIQUE ÉCONOMIQUE ENVIRONNEMENTALE}

Le développement des échanges, souvent associé à la croissance et à l'amélioration de la productivité, paraît généralement faire peu de cas de la protection de l'environnement. L'article 2, de la vérsion consolidée de 2002, concernant le Traité de Rome, du 25 mars de 1957, instituant la Communauté européenne (CE) :

L'Union se donne pour objectifs : de promouvoir le progrès économique et social ainsi qu'un niveau d'emploi élevé, et de parvenir à un développement équilibré et durable, notamment par la création d'un espace sans frontières intérieures, par le renforcement de la cohésion économique et sociale et par l'établissement d'une union économique et monétaire comportant, à terme, une monnaie unique, conformément aux dispositions du présent traité.

Cet article pourrait être interprété comme signifiant que les libertés de circulation sont des moyens alors que la protection de l'environnement est un objectif, ce qui conduirait à affirmer, contrairement aux idées reçues, la supériorité de cette dernière sur les libertés de circulation.

Lamarque (1973, p. XI) écrivait :

[...] la pollution des eaux et la pollution de l'air ne sont pas irrémédiables ; le bruit peut être contenu ; la dégradation des sites naturels, la réduction des espaces verts ou forestiers, la disparition d'espèces animales ou végétales ne présentent aucun caractère de fatalité : tout dépend de l'homme.

Selon Boutillier (2003), s'il nous paraît encore possible, plus de trente ans après, de faire preuve d'un certain optimisme et de considérer qu'il n'est pas trop tard pour préserver, voire améliorer, la qualité de l'environnement, il est cependant difficile d'ignorer le caractère parfois irréversible des dégâts occasionnés par le développement économique.

Le développement des échanges commerciaux n'est pas nécessairement contraire à la protection de l'environnement. Cependant il doit être observé que le développement des échanges commerciaux est plus souvent conçu comme un obstacle à la préservation de l'environnement qu'un facteur concourant à la réalisation de ce dernier objectif. En effet, l'interdiction de faire échec à la règle de la liberté des échanges pour des motifs environnementaux, revient à contraindre les Etats parties, signataires de traites commerciaux à abaisser leurs standards de protection au profit de la réduction des entraves tarifaires et des contraintes engendrées par des conventions (non tarifaires). Les démarches internes de chaque pays nous amènent à l'imposition de certaines contraintes au niveau global. Il suffit 
de constater qu'en raison de la nocivité de certaines méthodes de production, de la dangerosité de nombreux produits (l'exemple de la nouvelle réglementation européenne sur les substances chimiques appelée REACH - enRegistrement, Evaluation et Autorisation des substances CHimiques), la libre circulation des marchandises est de plus souvent primée par des considérations environnementales.

Il est difficile de délimiter les contours de la notion d'environnement. Ainsi, il n'existe pas une mais plusieurs acceptations de l'environnement, si bien qu'il est généralement admis qu'il s'agit là d'une " notion caméléon » (PRIEUR, 2004, p.1) variant en fonction des textes dans lesquels elle est définie. Passons à l'analyse économique au niveau interne de la politique environnementale.

\subsubsection{Au niveau interne}

Si la décision d'une action face aux changements climatiques n'est pas facile, ne rien faire maintenant, en attendant de nouvelles avancées de la science, ou un improbable miracle, est d'ores et déjà prendre le pari qu'aucune catastrophe majeure ne surviendra à l'avenir. C'est à l'évidence un pari terrible, dont les gagnants ou perdants ne sont pas nécessairement nous-mêmes, mais nos propres enfants ou petits-enfants, qui actuellement n'ont pas voix au chapitre, et ne pourront qu'assister impuissants, si un bouleversement gravissime survient, aux conséquences de nos actes actuels.

Malgré l'utilisation du terme « loi », l'économie, c'est-à-dire le système représentant nos échanges et leur valeur monétaire, n'est pas l'égale de la physique ou de la chimie, gouvernées par des règles que nous avons découvertes mais non décidées : avec ou sans hommes, la loi de l'attraction universelle ou la valeur de l'atome de carbone restent les mêmes. Par contre, ni un marché financier, ni une règle économique n'ont d'existence hors de notre présence : ils sont nés et disparaîtront avec nous (JANCOVICI, 2002, p. 122).

La première des conventions de prix indique que l'économie ne prend en compte que ce qui s'échange par la main de l'homme. N'est pas dans le champ de l'économie ce qui ne fait l'objet d'aucun échange de notre fait et n'a donc pas de prix. L'air que nous respirons, la pluie, le soleil, le vent, tout cela nous est donné sans que l'homme n'intervienne, et ces biens, au sens premier de cette expression, n'ont donc pas de prix. Dès à présent, on peut comprendre que cela peut rendre toute tentative de monétarisation 
des conséquences d'un dérèglement climatique un peu hasardeuse : cela revient à décider d'attribuer un prix à des choses qui ne peuvent se vendre ou s'acheter.

La seconde convention d'importance que nous avons prise, dans la majorité des pays du monde, est couramment désignée sous le terme « d'économie de marché ». Le prix d'un même bien, fabriqué de la même manière, par les mêmes individus, peut varier en fonction de l'offre et de la demande. De ce fait, un prix ne reflète pas seulement la valeur intrinsèque de production d'un bien, mais aussi le nombre de gens qui souhaitent en disposer et l'offre du moment.

La troisième convention stipule que la théorie économique ne connaît que des phénomènes parfaitement réversibles. Si j'échange mon argent contre du pain, puisque je revends mon pain pour la même somme d'argent, dans le monde économique je reviens à l'état initial. Il n'en va pas ainsi dans le monde réel : dans l'intervalle, j'ai perdu ne serait-ce que du temps, outre l'énergie utilisée pour me déplacer vers le lieu de vente et le lieu d'achat, etc. Ce principe de réversibilité des échanges pourrait à lui seul justifier que l'économie est une approximation limitée du monde réel, à manier avec la plus grande prudence : l'économie repose entièrement sur la violation de la deuxième loi de la thermodynamique (elle indique que les processus naturels sont fondamentalement irréversibles).

Enfin la dernière convention importante précise que disposer d'un bien dans le futur vaut moins cher que d'en disposer tout de suite.

L'économie est un mot qui comporte l'idée de réduction, dans le sens d'épargner, d'éviter de trop consommer. Une seconde signification, qui est même couramment acceptée, désigne les acteurs responsables des activités productives de l'homme. Les entreprises qui produisent des biens et services, les chaînes d'hypermarchés ou les agriculteurs font partie de cette économie-là.

Passons à l'analyse plus approfondie de la protection de l'environnement et de certaines démarches économiques liées à notre quotidien de consommateur.

\subsubsection{L'approche de la protection de l'environnement et du libre échange}

La préservation de l'environnement entretient des relations étroites avec la protection des consommateurs qui constitue une exigence impérative d'intérêt général. Le consommateur a en effet tout intérêt à bénéficier d'un environnement sain dans la mesure où ce dernier lui assure 
une certaine qualité de vie qu'il est en droit de revendiquer. La conscience environnementale des consommateurs s'est d'ailleurs développée au cours de ces dernières années, ce qui explique le recours de plus en plus étendu aux instruments fiscaux qui permettent de protéger l'environnement en incitant le consommateur à modifier son comportement. Il convient cependant de ne pas oublier que c'est la société de consommation qui est à l'origine de nombreuses atteintes à l'environnement, le consommateur souhaitant disposer d'une large gamme de produits à des prix compétitifs, sans nécessairement se soucier de la manière dont ces derniers ont été fabriqués. Par ailleurs, il est généralement admis que l'augmentation des déchets est essentiellement due aux habitudes de consommation, ce qui démontre que les intérêts du consommateur divergent très souvent de ceux qui doivent être sauvegardés au titre de la protection de l'environnement.

En définitive, il nous semble permis de considérer que la notion d'environnement comprend les éléments abiotiques comme l'air, l'eau et les sols, qui doivent être protégés à l'encontre de l'épuisement des ressources naturelles ou de la dégradation liée à des méthodes de production et des habitudes de consommation, ainsi que les éléments biotiques, c'est-à-dire les espèces vivantes, dont la protection passe par la conservation de la biodiversité et la préservation des habitats naturels.

Les standards nationaux de protection sont extrêmement différents d'un Etat à l'autre, ce qui explique les difficultés auxquelles se trouvent confrontés les opérateurs économiques lorsqu'il s'agit d'accéder à un marché étranger.

Les mesures de protection de l'environnement peuvent prendre de multiples formes, l'engouement pour les réglementations classiques conduisant généralement à adopter des mesures interdisant ou restreignant la production, la mise sur le marché ou l'emploi de certains produits ayant des effets négatifs sur l'environnement. A côté de ce type de réglementations, on a de plus en plus souvent recourus aux instruments économiques, particulièrement les instruments fiscaux, qui visent à inciter le producteur et le consommateur à privilégier les méthodes de fabrication et les produits respectueux de l'environnement.

La marchandise peut être considérée, selon l'arrêt du 10 décembre 1968, concernant l'affaire 7/68, de la Commission des communautés européennes contre la République italienne, par la Cour de Justice des Communautés Européennes (CJCE, 1968, p. 626) comme « les produits appréciables en argent et susceptibles, comme tels, de former l'objet de 
transactions commerciales », ce qui implique que la plupart des objets et des substances dangereuses pour l'environnement sont soumis au respect de l'interdiction des entraves aux échanges.

Il apparaît qu'il est extrêmement difficile de concilier la libre circulation des marchandises vers la liberté des consommateurs et la préservation de l'environnement. Ainsi, la recherche d'un compromis satisfaisant est « une question de société d'autant plus délicate qu'elle oppose, peu ou prou, les intérêts du patrimoine commun, qui sera légué aux générations suivantes, aux considérations économiques immédiates » qui sont encore très présentes au sein de la Communauté européenne (THIEFFRY, 1998, p. 244).

La libération des échanges, loin de présenter un caractère original, est constamment affirmée comme un objectif que les Etats doivent impérativement toucher tant sur le plan national que sur le plan international.

L'interdiction des entraves aux échanges et l'instauration d'un marché intérieur sont en définitive deux objectifs communautaires qui se complètent et s'enrichissent l'un à l'autre. En effet, si la libre circulation est une condition fondamentale de l'existence d'un marché unique européen, l'achèvement de cet espace sans frontières intérieures a pour nécessaire conséquence de faciliter les échanges de marchandises entre les Etats membres, les opérateurs économiques se comportent finalement de la même manière que dans le cadre d'un marché interne où le principe de la liberté du commerce et de l'industrie va de soi.

La Commission des Communautés européennes a défini le contenu du concept de "marché intérieur unique ", à l'occasion de la présentation de son Livre blanc à l'intention du Conseil Européen (1985, p. 2), comme un marché « libre où les biens, les personnes, les services et les capitaux circuleraient en toute liberté, et dont le bon fonctionnement serait assuré par des garanties contre les distorsions de concurrence, par le rapprochement des législations et, enfin, par l'harmonisation de la fiscalité indirecte ».

Si la liberté des échanges est un objectif fondamental, elle nécessite pour être réalisée une élimination aussi complète que possible des difficultés au commerce et la considération pour d'autres intérêts qui ne sont pas d'ordre économiques qui méritent d'être pris ne compte. Si la sauvegarde de certains intérêts supérieurs à la suppression des entraves aux échanges est légitime, leur prise en considération ne doit malgré tout pas conduire à un affaiblissement du principe de la libre circulation des marchandises. Le caractère fondamental de ce principe implique ainsi que les dérogations 
qui peuvent être accordées dans certains cas soient d'interprétation stricte, tant en ce qui concerne leur délimitation que l'application des conditions auxquelles elles sont soumises.

L'article $30^{6}$ du Traité CE présente une énumération des intérêts protégés. Son article $95, \S 4^{7}$ permet aux Etats, sous certaines conditions, de maintenir des dispositions nationales contraignantes, même si une mesure d'harmonisation est adoptée.

Ces différents objectifs non économiques qui ont nettement gagné en importance depuis les débuts de la construction communautaire, sont dès lors susceptibles de primer la libre circulation des marchandises. C'est le cas de la protection de l'environnement. La confrontation, des objectifs de protection de l'environnement et de libre circulation des marchandises, est inévitable dans les systèmes qui dépassent le niveau national.

Le but de la dérogation prévue à l'article $95, \S 4$ à 9 , du traité est en effet de préserver l'environnement aussi efficacement que possible sans remettre en cause la libre circulation des marchandises.

\section{Le Traité d'Amsterdam ${ }^{8}$ est venu confirmer l'obligation de motivation}

6 Ex article $36 \mathrm{du}$ Traité : « Les dispositions des articles 30 à 34 inclus ne font pas obstacle aux interdictions ou restrictions d'importation, d'exportation ou de transit, justifiées par des raisons de moralité publique, d'ordre public, de sécurité publique, de protection de la santé et de la vie des personnes et des animaux ou de préservation des végétaux, de protection des trésors nationaux ayant une valeur artistique, historique ou archéologique ou de protection de la propriété industrielle et commerciale. Toutefois, ces interdictions ou restrictions ne doivent constituer ni un moyen de discrimination arbitraire, ni une restriction déguisée dans le commerce entre les États membres ».

7 Ex article $92:$ «1. Sauf dérogations prévues par le présent traité, sont incompatibles avec le marché commun, dans la mesure où elles affectent les échanges entre États membres, les aides accordées par les États ou au moyen de ressources d'État sous quelque forme que ce soit, qui faussent ou qui menacent de fausser la concurrence en favorisant certaines entreprises ou certaines productions. 2. Sont compatibles avec le marché commun : a) les aides à caractère social octroyées aux consommateurs individuels, à condition qu'elles soient accordées sans discrimination liée à l'origine des produits ; b) les aides destinées à remédier aux dommages causés par les calamités naturelles ou par d'autres événements extraordinaires ; c) les aides octroyées à l'économie de certaines régions de la république fédérale d'Allemagne affectées par la division de l'Allemagne, dans la mesure où elles sont nécessaires pour compenser les désavantages économiques causés par cette division. 3. Peuvent être considérées comme compatibles avec le marché commun : a) les aides destinées à favoriser le développement économique de régions dans lesquelles le niveau de vie est anormalement bas ou dans lesquelles sévit un grave sous-emploi ; b) les aides destinées à promouvoir la réalisation d'un projet important d'intérêt européen commun, ou à remédier à une perturbation grave de l'économie d'un État membre ;c) les aides destinées à faciliter le développement de certaines activités ou de certaines régions économiques, quand elles n'altèrent pas les conditions des échanges dans une mesure contraire à l'intérêt commun. Toutefois, les aides à la construction navale existant à la date du 1er janvier 1957, pour autant qu'elles ne correspondent qu'à l'absence d'une protection douanière, sont progressivement réduites dans les mêmes conditions que celles applicables à l'élimination des droits de douane, sous réserve des dispositions du présent traité visant la politique commerciale commune vis-à-vis des pays tiers ; d) les autres catégories d'aides déterminées par décision du Conseil statuant à la majorité qualifiée sur proposition de la Commission ».

8 Traité d'Amsterdam modifiant le Traité sur l'Union Européenne, les Traités instituant les Communautés Européennes et certains actes, signé le 2 octobre 1997, est entré en vigueur le 1er mai 1999. 
en indiquant qu'il revenait à l'Etat membre d'expliquer « les raisons » pour lesquelles il estime nécessaire d'appliquer des mesures environnementales plus restrictives que celles prévues par le réglementation communautaire, la notification devant ainsi faire apparaitre " tout argument scientifique susceptible d'étayer le bien-fondé du niveau de protection » envisagé. L'article $95 \mathrm{du}$ traité reste silencieux en ce qui concerne les conséquences d'une insuffisance de motivation de la notification.

Ayant développé l'analyse de la protection de l'environnement face au libre échange, étudions les démarches organisationnelles vers la protection de l'environnement.

\subsubsection{Les démarches organisationnelles}

L'engagement environnemental des organisations peut être identifié comme une réponse pro active face aux pressions externes. Les efforts environnementaux des organisations vers la réduction des ses émissions de GES peuvent également être interprétés à partir de la prise en compte plus ou moins volontaire de la problématique des changements climatiques. Cette prise en compte est liée à la perception des opportunités ou des risques économiques associés au Protocole de Kyoto et des mesures dans ce domaine.

Ainsi, si la réduction des GES est perçue comme une source d'économies et de productivité, il est possible de supposer que les dirigeants seront davantage incités à adopter une politique engagée et à planifier des investissements significatifs pour réduire leurs émissions. Si une telle démarche est perçue comme une source de coûts et de désavantages compétitifs, les dirigeants seront plutôt enclins à résister à ce type de politique et à adopter une réponse plus passive ou plus réactive par rapport à l'émergence de contraintes dans ce domaine.

Un second axe important pour définir la réponse des organisations par rapport aux changements climatiques est l'intensité des pressions externes pour réduire les émissions de GES.

Selon Boiral (2006), ces pressions sont surtout centrées sur les grands émetteurs industriels, qui concentrent une proportion importante des émissions globales liées à l'activité humaine. Cependant, la problématique des changements climatiques ne concerne pas seulement les grands émetteurs industriels. D'une part, les autres organisations représentent, de façon cumulée, une proportion significative des émissions totales de GES. D'autre 
part, nombre de ces organisations peuvent être concernées à des degrés différents par cette problématique même en l'absence de pressions externes à ce sujet: vente d'équipements de mesure ou de contrôle des émissions, conseils environnementaux, activités forestières contribuant à stocker des GES, entreprises souhaitant se donner une image verte, etc.

En croisant les deux dimensions précédemment décrites, soit la stratégie plus ou moins engagée concernant les enjeux des changements climatiques et l'intensité des pressions externes à ce sujet, il est possible de distinguer quatre principaux types de réponses organisationnelles concernant cette problématique : la réponse passive, la réponse défensive, la réponse proactive et la réponse promotrice.

La réponse passive correspond à un statu quo par rapport aux enjeux des changements climatiques de la part d'organisations qui ne sont pas l'objet de fortes pressions à ce sujet. La plupart de ces organisations ne sont pas considérées comme de grands émetteurs industriels. Dans cette perspective, les émissions de GES et la signature du Protocole de Kyoto n'apparaissent pas comme des menaces ou des opportunités immédiates et significatives. Ces enjeux ne sont donc pas réellement reconnus et pris en compte dans la stratégie corporative pour autant qu'ils ne menacent pas directement les activités habituelles. Les organisations qui adoptent une réponse passive n'ont généralement pas mis en œuvre de systèmes de gestion environnementale ou n'ont pas pris en compte la réduction des GES dans ce système. La principale raison de ce type de réponse est la politique du «business as usual - BAU ». En effet, si les organisations ne sont pas l'objet de pressions externes et ne sont pas perçues comme de grands émetteurs, la remise en cause des habitudes peut paraître superflue. Cette approche passive, semble d'autant plus légitime que la croyance de la réduction des GES entraîne des coûts significatifs, est assez largement partagée.

Dans ce contexte, beaucoup d'organisations tendent à maintenir le statu quo et ne réagissent à cette problématique que si elles y sont véritablement contraintes. Cependant, cette réponse passive tend à ignorer la montée des pressions internationales pour la réduction des GES et le renforcement prévisible des normes dans ce domaine. Si les pressions sont aujourd'hui centrées sur quelques grandes entreprises polluantes, il est probable qu'elles vont également concerner, à plus ou moins brève échéance, d'autres organisations. Dans cette condition, la distinction intermédiaire est prise en compte dans la stratégie corporative pour autant qu'elle ne menace pas directement les activités habituelles. 
Une autre situation émane des fortes pressions à l'encontre des entreprises les plus polluantes. Ces pressions ne se traduisent pas nécessairement par une réaction défensive. Plutôt que de s'opposer plus ou moins ouvertement au protocole de Kyoto, certains grands émetteurs de GES reconnaissent au contraire la nécessité de réduire leurs émissions et ont pris des engagements significatifs en la matière en anticipant l'émergence de contraintes externes. Ainsi, la réponse proactive correspond mieux à des pressions externes fortes et à un soutien actif aux mesures pour réduire les émissions de GES.

Contrairement aux entreprises qui ont adopté une position plus défensive, les institutions proactives soulignent souvent les avantages économiques des mesures pour réduire les GES. Ces engagements environnementaux témoignent d'une stratégie à long terme pour différencier l'entreprise par rapport à des concurrents qui adoptent une position plus défensive et pour anticiper la montée des pressions à l'encontre des grands émetteurs industriels.

Le dernier type d'attitude, la réponse promotrice, correspond à une approche proactive de la part d'organisations qui ne font pas partie d'objet de fortes pressions externes pour réduire leurs émissions de GES. N'étant généralement pas considérées comme de grands émetteurs industriels, ces organisations cherchent rarement à répondre à des menaces externes clairement définies. Leurs motivations sont plutôt de nature commerciale, stratégique, socioéconomique ou éthique. Ces motivations relèvent donc d'une démarche de promotion volontaire plus que d'une anticipation de contraintes perçues comme plus ou moins imminentes. Le premier aspect qui peut inciter ces organisations à s'engager dans cette démarche est lié à l'émergence de nouveaux marchés. Par exemple, en développant une expertise unique dans le domaine de l'énergie éolienne sur le marché domestique, les organisations danoises de ce secteur comme Vestas sont devenues les leaders mondiaux de cette industrie en pleine croissance. La mise en œuvre d'un marché international d'échanges de CO2 a également favorisé le développement d'organisations spécialisées dans ce type de transactions, comme EcoSecurities ou encore NatSource. De même, la prise en compte des « puits de carbone » dans le Protocole de Kyoto encourage les activités de reboisement pour compenser les émissions de GES. Par exemple, la société anglaise Future Forest s'est spécialisée dans cette activité et propose aux organisations des services pour évaluer leurs émissions de carbone et les compenser à travers le financement de divers projets dans le secteur 
forestier. Pour d'autres organisations, la promotion de projets visant à réduire les GES représente un moyen de se différencier de la concurrence en se donnant une image et une vocation verte ou éthique.

Ces quatre principaux types de réponses aux enjeux du réchauffement climatique ne sont pas statiques et monolithiques. En effet, la complexité et l'actualité de ces enjeux sont susceptibles de modifier assez rapidement les pressions internationales et les politiques dans ce domaine. Ces changements peuvent affecter plus ou moins directement la stratégie nationale des organisations et la pertinence de l'une ou l'autre des réponses face à la problématique des émissions de GES. Par exemple, certaines organisations hésitent à mettre en œuvre des mesures proactives en faveur du Protocole de Kyoto en l'absence de politiques publiques claires à ce sujet. Une des craintes est que les efforts entrepris ne soient pas reconnus par la suite et que les organisations soient contraintes de réaliser des investissements supplémentaires ne tenant pas compte des progrès déjà accomplis. Les incertitudes sur les politiques publiques en faveur du protocole de Kyoto peuvent donc favoriser une réponse passive ou défensive par les organisations et encourager un certain attentisme. Cependant, cet attentisme repose sur une logique « gain/perte », de l'anglais «win-loose » qui prend pour acquis que la réduction des émissions de gaz à effet de serre représente des coûts qu'il est préférable d'éviter ou de retarder en l'absence de pressions externes clairement définies. De plus, il ne tient pas compte des avantages concurrentiels qui peuvent découler de l'introduction de normes ou de politiques plus sévères en faveur de la réduction des émissions de GES. En toute hypothèse, la mise en place de ces normes et de ces politiques conduit à remettre en cause la politique de l' « attendre et voir », de l'anglais « wait and see ». En fait, tout porte à croire que les organisations, quels que soient les secteurs d'activités sont de plus en plus amenées, de façon plus ou moins volontaire, à modifier leur politique environnementale et à prendre des engagements plus significatifs en faveur de la réduction des GES. Pour beaucoup d'organisations, ces engagements supposent le passage d'une réponse de type défensive ou passive à une réponse de type proactive ou promotrice. Cette transition vers une stratégie proactive, c'est-à-dire reposant sur un engagement volontaire et significatif en faveur de la réduction des GES et du soutien au protocole de Kyoto se justifie par trois aspects interdépendants : les pressions institutionnelles croissantes, les impacts sur l'avantage concurrentiel des organisations et les bénéfices économiques découlant d'une telle démarche. 
La montée des pressions institutionnelles pour la réduction des émissions de GES représente une des raisons pour justifier la mise en œuvre d'une stratégie proactive dans ce domaine. En effet, comme l'ont montré les approches néo institutionnelles, la quête de légitimité sociale représente un ressort fondamental du changement organisationnel, en particulier dans le domaine de la gestion environnementale. Cette quête tend à favoriser le développement de pratiques et de politiques similaires, conduisant les organisations à devenir plus iso morphiques afin de répondre aux attentes de la société. Ce souci de conformité et de légitimité l'emporte souvent sur les préoccupations d'efficience économique.

Dans ce contexte, les organisations qui adoptent une réponse défensive ou passive par rapport au Protocole de Kyoto en invoquant, notamment, des arguments économiques sont de plus en plus exposées à des critiques et à des remises en causes qui peuvent compromettre leur légitimité voire leur pérennité. Ces positions semblent aujourd'hui à contre-courant, tant par rapport aux attentes de la société que par rapport aux positions adoptées par un nombre croissant d'organisations. En effet, le réchauffement climatique et le Protocole de Kyoto ne constituent plus des enjeux théoriques ou hypothétiques préoccupant surtout les environnementalistes. L'entrée en vigueur, notamment en Europe, de mesures comme la mise en place de quotas d'émissions et de permis échangeables, affecte dès à présent de nombreuses organisations, y compris étrangères à travers des filiales implantées dans les régions concernées par ces mesures. De plus, les politiques environnementales des États peuvent changer rapidement, et un pays qui n'a pas ratifié le Protocole de Kyoto peut décider de le faire comme ce fut le cas avec la Russie en novembre 2004 et l'Australien en 2007. Enfin, il est probable que ces politiques vont s'élargir et ne plus concerner seulement les grands émetteurs. Il est donc raisonnable d'anticiper une augmentation croissante des pressions environnementales à l'encontre des grands comme des petits émetteurs de GES, rendant les réponses défensives et passives de moins en moins légitimes (BOIRAL, 2005, p. 1-9).

L'application du principe de précaution, qui est aujourd'hui accepté par de nombreux pays, notamment en Europe, est une première raison qui entreprend, en faveur de la mise en œuvre, une stratégie proactive. La seconde raison qui milite en faveur de cette stratégie est liée aux avantages concurrentiels qu'une telle démarche peut apporter. Ces avantages découlent d'abord de la constitution de barrières environnementales favorisant les organisations les moins polluantes. En effet, ces organisations sont en meilleure position pour répondre à la montée des pressions externes liées au réchauffement climatique que des concurrents qui ont adopté une position plus défensive ou passive et qui pourront plus difficilement se conformer aux nouvelles exigences environnementales. En outre, l'adoption d'une 
stratégie proactive permet d'anticiper de façon plus souple les pressions externes et de maintenir la marge de manœuvre de l'organisation.

Ces avantages rejoignent la théorie du cycle de vie des pressions sociétales, qui montre que l'autonomie des organisations tend à diminuer au fur et à mesure que les pressions externes se renforcent. Ce phénomène peut expliquer en partie la constitution de coalitions d'entreprises se regroupant pour se doter de mesures volontaires d'autocontrôle des émissions de GES afin de mieux contrôler ou d'éviter l'émergence de règlements trop stricts en la matière. En France par exemple par le Mouvement des entreprises de France (MEDEF) est la principale organisation d'entreprises françaises à négocier avec le gouvernement des ententes volontaires pour gérer les quotas et les permis d'émissions de $\mathrm{CO} 2$ mis en œuvre en Europe à partir de janvier 2005. Selon Boiral (2004), ce type d'entente environnementale n'est pas nouveau. Il s'est rapidement développé à partir des années 80 avec la remise en cause des approches réglementaires traditionnelles dans le domaine de l'environnement. Pour les organisations, les ententes volontaires et l'anticipation des normes environnementales ne permettent pas seulement de mieux se préparer ou de mieux contrôler les changements en cours. Elles permettent aussi d'imposer aux concurrents moins proactifs des normes qui rendent plus difficile l'entrée sur certains marchés. De même, ce type de stratégie proactive peut être observé aujourd'hui par des organisations qui ont réalisé des investissements majeurs afin de réduire leurs GES et qui soutiennent les politiques publiques en faveur du Protocole de Kyoto.

La dernière motivation en faveur du développement d'une stratégie proactive est liée aux bénéfices économiques d'une telle démarche. Ces bénéfices ne découlent pas seulement de la logique "gain/gain », de l'anglais «win-win », inhérente à certaines actions environnementales. Elle résulte également des conséquences de la mise en œuvre des politiques publiques dans le cadre de la ratification du Protocole de Kyoto. Outre les aides directes sous forme de subventions ou de crédits d'impôts qui se développent progressivement, la création d'un marché d'échange de permis d'émissions de GES peut avoir un impact économique significatif, en particulier pour les grands émetteurs industriels. En effet, ce type de marché permet d'appliquer le principe pollueur payeur. Ainsi, les organisations qui ne respectent pas leurs quotas d'émissions devront à terme faire l'acquisition de permis d'émissions sur les marchés internationaux pour compenser leurs mauvaises performances environnementales. En 
revanche, les organisations qui auront réussi à réduire leurs émissions en dessous des quotas fixés pourront vendre de tels permis et en tirer un avantage économique non négligeable.

La même remarque s'applique au niveau des États, qui pourront également acheter ou vendre des permis d'émissions de GES. Ce type de marché ne se développe pas seulement en Europe.

L'adoption d'une stratégie proactive favorise, à plus ou moins long terme, des bénéfices sur ce type de marché indépendamment des impacts économiques découlant directement des mesures de dépollution entreprises. Cette stratégie permet également de limiter certains risques financiers. D'une part, les performances environnementales sont de plus en plus un critère d'évaluation sur les marchés financiers et contribuent à l'appréciation de la bonne gouvernance de l'organisation. D'autre part, le secteur des banques et des assurances accorde de plus en plus d'importance à ces aspects.

Enfin, la mise en œuvre d'une stratégie proactive peut être un puissant moyen de mobiliser l'ensemble du personnel (national ou international) autour d'enjeux globaux qui transcendent les frontières organisationnelles. Cette mobilisation est essentielle pour réduire de façon efficiente les impacts environnementaux des activités de l'organisation. Elle favorise également la fierté, la participation et la mobilisation du personnel, facteurs déterminants pour améliorer la productivité.

Elle s'applique également aux processus stratégiques en général (la nature des décisions et des plans) pour concrétiser l'adoption d'une stratégie pro active qui dépend des secteurs d'activités et des spécificités de chaque organisation. Cependant les organisations doivent plus considérer le Protocole de Kyoto comme une opportunité que comme une menace ou un coût.

En premier lieu, avec l'entrée en vigueur du protocole, les opportunités et les menaces sont plus immédiates, même si de nombreuses incertitudes demeurent au sujet des politiques publiques et aux pressions environnementales à venir. En deuxième lieu, les organisations doivent faire un inventaire aussi précis que possible de leurs émissions de GES. Cet inventaire est nécessaire pour deux raisons principales. La première raison est qu'il permet de mieux connaître les principales sources d'émissions et donc de mieux cibler les mesures à mettre en œuvre. La seconde, est qu'il permet de mesurer les performances dans ce domaine et de participer éventuellement aux échanges de permis d'émissions. La mesure et la 
surveillance des émissions de GES sont d'ailleurs indispensables à la mise en place du " système d'observance » prévu par la Conférence de Bonn de 2001. En troisième lieu, pour être crédibles, les dirigeants doivent définir des politiques et des objectifs clairs dans ce domaine.

La mise en œuvre d'objectifs de réduction des émissions de GES plus prétentieux dépend de la nature plus ou moins proactive de la stratégie environnementale de l'organisation et des opportunités ou menaces qui peuvent en découler. La mise en place de plans, de politiques et de mécanismes de mesure des performances peut être intégrée dans un système de gestion environnementale de type ISO 14 001. La même remarque s'applique pour d'autres mesures telles que la formation du personnel, l'identification des sources de rejet des GES, la rédaction de procédures environnementales, ou encore la définition des rôles et des responsabilités. Cette démarche de certification permet d'améliorer la rigueur des programmes environnementaux tout en apportant une reconnaissance externe des efforts entrepris dans ce domaine. En dernier lieu, nombre d'organisations devront investir davantage dans des programmes de recherche et développement en environnement. Étant donné les coûts que peuvent représenter ces programmes, les partenariats avec d'autres organisations sont appelés à se développer.

\section{CONCLUSION}

Les catastrophes environnementales récentes révèlent de plus en plus de répercussions économiques majeures, susceptibles de secouer la santé, les finances et le marché du travail, même dans les pays économiquement développés, comme les États-Unis. L'application de la théorie des jeux en tant que méthode d'analyse, de problématisation et de résolution des problèmes environnementaux, plus que jamais, s'avère pertinente et adéquate dans le scénario actuel, dont les problèmes dépassent les frontières nationales et provoquent des catastrophes aux grandes répercussions dans les économies nationales. Les possibilités aléatoires de pertes par tous les participants au jeu au niveau mondial révèlent que seule l'adoption d'une politique sérieuse et coopérative entre les États nationaux, visant à orienter les secteurs économiques vers la durabilité environnementale, peut contenir ou éviter (totalement ou partiellement), avec une plus grande efficacité, la répétition de catastrophes majeures et de répercussions transfrontalières.

La dimension substantiellement universelle de l'idée d'environnement 
entraîne une grande difficulté à définir ses limites et son contenu concret, ce qui a pour conséquence de créer de nombreuses difficultés pour traduire les demandes de protection et relatives en termes juridiques.

C'est une question très complexe, à laquelle il convient de ne faire ici qu'une brève référence, qui met en crise la manière de concevoir les systèmes juridiques sur la base d'une approche fondée sur la tradition du droit positif (JONAS, 1979, p. 8): comme nous l'avons vu à cet égard en fait, le problème des réfugiés environnementaux est très difficile à définir : quels sujets devraient être considérés comme «titulaires» du droit à l'environnement, car c'est quelque chose qui affecte tout le monde. De même qu'il est difficile de définir, dans un sens objectif, ce qui doit être compris comme le «contenu» de la notion d'environnement, car l'»environnement» représente tout ce qui nous entoure et parce que nous ne prenons conscience de la «valeur de l'environnement» que lorsque nous percevons une réduction ou dommages.

Précisément, ces deux caractéristiques entraînent une grande difficulté à traduire l'intérêt général pour la protection de l'environnement en un cas juridique bien défini, qui peut représenter une référence efficace pour constituer un système de protection juridiquement structuré, car avoir un cas normatif de référence est toujours nécessaire dans le contexte du droit positif contemporain.

Pour ces raisons, le développement d'une protection juridique de l'environnement a dû recourir à un chiffre très particulier, représenté par le principe de précaution (STEEL, 2015): cela permet de donner accès à l'environnement dans la dimension de systèmes juridiques positifs en tant que «bien juridique», permettant de le protéger et aussi de le comparer avec d'autres «atouts juridiques», comme la concurrence et les échanges économiques, dans le but de pouvoir équilibrer la protection de tous ces «atouts juridiques».

Le principe de précaution y réussit car il permet, sur le plan juridique, de donner de l'importance à l'environnement par rapport à ses éventuels dommages et d'essayer de les anticiper: si, en effet, il est vrai que la «valeur de l'environnement» devient matériellement appréciable lorsque il perd une partie de l'environnement lui-même, il est également vrai que souvent ces pertes, ou dommages, ne sont plus récupérables.

À ce stade, cependant, le problème se pose de donner un contenu défini du risque de dommages à l'environnement, afin de définir l'objet et les destinataires de ces règles de protection (SUSTEIN, 2015, p. 1003) : sans 
établir « qui, quand et quoi » est obligatoire de faire dans termes juridiques on ne peut pas prévoir de véritables règles juridiques, mais seulement prévoir de faibles affirmations d'intention qui sont valables en tant que soft law.

De nombreuses dispositions contenues dans les règles du droit international relatives à la protection de l'environnement ne dépassent pas le niveau de simples déclarations de principes directeurs, sans pour autant être dotées d'une efficacité réellement contraignante.

La raison, en résumé, est que l'exercice d'une activité de règlement des litiges au cas par cas permet également de mettre en œuvre concrètement des règles qui ont du mal à être pleinement affirmées au niveau général et politique, car elles se réfèrent à des intérêts trop généraux. et diffusés pour être immédiatement identifiés en termes objectifs et subjectifs, comme cela se produit pour la protection de l'environnement et en marge de la prévisibilité scientifique de la certitude de l'occurrence de dommages à l'environnement (O'RIORDAN; CAMERON; JORDAN, 2001).

En d'autres termes, c'est précisément l'activité de juridiction, liée à l'application des règles de «responsabilité juridique» pour l'exercice d'activités jugées dangereuses (SALEILLES, 1897; JOSSERAND, 1937), que de permettre aux contours subjectifs et objectifs de la protection de l'environnement de s'établir progressivement, à travers l'indication du "quand» et du «comment» appliquer le principe de précaution pour équilibrer la protection de l'environnement et d'autres intérêts pertinents, tels que les besoins du commerce international ou la concurrence sur le marche (MIGLIORINI, 1997, p. 677; FERRARA, 2003, p. 526; PORCHIA, 2014, p. 155).

Sur la base de ce que les juges ont décidé précédemment, condamnant ou absout l'accusation de causer des dommages et l'indemnisation y afférente, il devient possible d'identifier qui et ce qui est légalement obligatoire de faire ou de ne pas faire pour remplir les obligations de protection de l'environnement.

L'évolution ultérieure des règles de protection de l'environnement représente souvent la traduction de ces résultats des décisions des juges au niveau des règles préventives: éviter une nouvelle peine pour compenser les dommages causés à l'environnement constitue, en ce sens, la motivation qui motive à la fois les États individuels doivent prévoir des règles obligatoires dans leurs systèmes généraux, et les particuliers ou les entreprises doivent adopter eux-mêmes des règles d'autoréglementation. 


\section{BIBLIOGRAPHIE}

AQUILINA, K. AQUINTA, P. Il sistema ambiente tra etica, diritto ed economia. Padova: CEDAM, 2013.

BECK, U. World risk society. Cambridge: Cambridge University Press, 1988.

BIANCHI, A; GESTRI, M. Il princpio di precauzione nel diritto internazionale e comunitário. Milano: Giuffrè, 2006.

BOIRAL, O. Réduire les gaz à effet de serre: Tchernobyl économique ou indicateur de la compétence des dirigeants? Franc Vert, v. 2, n. 3, nov. 2005.

BOIRAL, O. Réchauffement climatique et stratégies d'entreprises. Les Cahiers de l'IHQEDS, v. 1, n. 2, déc. 2006.

BOUTILLIER, S. Les économistes et l'écologie, enseignements historiques. Innovations, n. 18, 2003.

COMMISSION DES COMMUNAUTÉS EUROPÉENNES. L'achèvement du marché intérieur, Livre blanc de la Commission à l'intention du Conseil européen. Bruxelles, 14 de juin 1985. Disponible en : http:// www.cvce.eu/obj/livre_blanc_sur_1_achevement_du_marche_interieur_14_juin_1985-fr-0d72b347-b235- 4c9d-bb71-ba38824f5d49. html. Accès en : 12 nov.2020.

COUR DE JUSTICE DES COMMUNAUTÉS EUROPÉENNES (CJCE). Affaire 7/68 Commission des communautés européennes contre République italienne. 10 déc. 1968. Disponible en : https://eur-lex.europa.eu/ legal-content/FR/TXT/PDF/?uri=CELEX:61968CJ0007\&from=FR. Accés en: 12 nov. 2020.

DEMARIA, C. Développement durable et finance. Paris: Maxima, 2004.

FAUCHEUX, S.; NOËL, J.-F. Economie des ressources naturelles et de l'environnement. Paris: Armand Colin, 1995.

FERRARA, R. I principi comunitari della tutela dell'ambiente. Diritto Ambientale, n. 3, 2003.

FRANCE. Ministére de L'Economie, des Finances et de la Relance. 
Industrie. Disponible en : https://www.entreprises.gouv.fr/fr/industrieentreprise. Acesso em: 12 nov. 2020.

FREESTON, D; HEY, E. The precautiorary principle and international law: the challenge of implementation. Kluwer: L'Aja, 1996.

GALLAIS, J. Les tropiques: terres de risques et de violences. Paris : Armand Collin, 1994.

JANCOVICI, J.-M. L'avenir climatique. Quel temps ferons-nous? Paris: Editions du Seuil, 2002.

JONAS, HANS. Das Prinzip Verantwortung. Frankfurt am Maine: Insel Verlag, 1979.

JOSSERAND, L. L'essor moderne du concept contractuel. Paris: Sirey, 1937.

LAFFITTE, P.; SAUNIER, C. Office Parlementaire d'évaluation des choix scientifiques et technologiques - Rapport sur Les apports de la science et de la technologie au développement durable, Tome I: Changement climatique et transition énergétique: dépasser la crise. Enregistré à la Présidence de l'Assemblée le 28 juin 2006.

LAMARQUE, J. Droit de la protection de la nature et de l'environnement. Paris: LGDJ, 1973.

LUHMANN, N. Soziologie des Risikos. Berlin: De Gruyter, 1991.

MEADOWS, D. H. et al. The limits to Growth. Boston: Massachussets Institute of Technology, 1972.

MIGLIORINI, L. Le eccezioni ambientali ai principi del GATT nella prassi dei PANELS. Diritto del Commercio Internazionale, n. 3, 1997.

O'RIORDAN, J; CAMERON, J; JORDAN, A. Reinterpretatig the precautionary approach. London: Cameron May, 2001.

PORCHIA, O. Le politiche dell'Unione Europea in materia ambientale. In: FERRARA, R.; GALLO, C. E. (a cura di). Trattato di diritto dell'ambiente. Milano: Giuffrè, 2014.

PRIEUR, M. Droit de l'environnement. 5. ed. Paris: Dalloz, 2004.

ROME. Traité de Rome, de 25 mars 1957. Traité instituant la Communauté européenne. Journal Officiel des Communautés Européennes.Vérsion 
consolidée, C 325/01, Rome [1957]. Disponible en : https://eur-lex.europa.eu/legal-content/FR/TXT/PDF/?uri=CELEX:12002E/TXT\&from=FR. Accès en: 12 nov. 2020.

SALEILLES, R. Les accidents de travail et la responsabilité civile: essai d'une théorie objective de la resaponsabilité délictuelle. Paris: Laussanne, 1897.

STEEL, D. Philosophy and the preacautionary principle: science, evidence and environmental policy. Cambridge: Cambridge Univerity Press, 2015.

SUSTEIN, C. R. Beyond the precautionary principle. University of Pennsylvania Law Review, v. 151, 2015.

THIEFFRY, P. Droit européen de l'environnement. Paris: Dalloz, 1998.

VENTURINI, G. L'Organizzazione Mondiale del Commercio. Milano: Giuffrè, 2015.

Reçu le 21/05/2020. Accepté le 22/10/2020.

\section{Cit cet article (ABNT):}

GARBACCIO, G. L.; FLACCO, R. J. La protection et la politique économique environnementales. Veredas do Direito, Belo Horizonte, v. 17, n. 39, p. 147-175, maio/ago. 2020. Disponible sur le site http://www.domhelder. edu.br/revista/index.php/veredas/article/view/1833. Accès en: jour mois. année. 